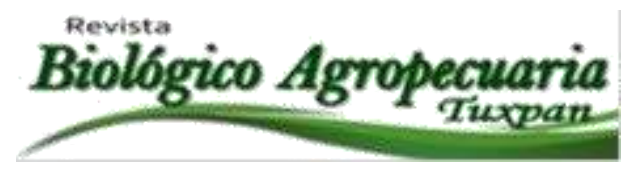

\title{
Estudio de mercado para determinar la demanda existente de huevo de codorniz en
}

Tuxpan, Veracruz

\begin{abstract}
Díaz Reyes Joaquín Eduardo ${ }^{1 ष}$; Mateos Rocha Roberto Alejandro ${ }^{1}$, Elorza Martínez Pablo ${ }^{1}$; Garcez Mercado Nora ${ }^{1}$, Edalid Álvarez Velázquez ${ }^{2}$, Sonia Reyes Reyes ${ }^{2}$, Blanca Vianey Hidalgo Barrios ${ }^{2}$ y Oralia Elorza Martínez ${ }^{2}$
\end{abstract}

${ }^{1}$ Facultad de Ciencias Biológicas y Agropecuarias de la Universidad Veracruzana, campus Tuxpan. ${ }^{2}$ Facultad de Contaduría de la Universidad Veracruzana, campus Tuxpan

${ }^{\bowtie}$ Autor para correspondencia: eduardo_3909@hotmail.com

Recibido: 15/08/2016
Aceptado: 19/11/2016

\section{RESUMEN}

El proyecto tiene como objetivo determinar la demanda de huevo de codorniz en la ciudad de Tuxpan, Veracruz, razón por la cual se inicia con una observación preliminar de la actividad en el municipio, mediante un estudio de tipo objetivo, determinándose que no existe mercadeo del huevo de codorniz. Se observa un bajo hábito de consumo de huevo de codorniz y por lo tanto se debe de concientizar a la población de los beneficios que este producto aporta a la salud así como el precio al que se pretende ofrecer para que de esta manera se penetre en el mercado poco a poco hasta llegar a posicionarnos. Asimismo, se requiere de amplias campañas de publicidad a nivel local, regional y nacional, para que las personas consuman un producto de alto nivel nutricional y por lo tanto, con grandes beneficios para cualquier tipo de población, incluidos niños, adultos mayores y personas enfermas. Todo esto se determinó por medio de una encuesta aplicada a la población para lograr nuestro principal objetivo, determinar si existe una demanda del producto en la región, llegando a la conclusión que efectivamente existe una demanda ya que el $98 \%$ de la población encuestada contesto que está de acuerdo en comprar el producto en cuanto este sea puesto a la venta. Esto también se debe a que la mayoría desconoce el producto y por lo tanto no está dispuesto a comprarlo debido a este desconocimiento, siendo importante posicionarlo en la mente del consumidor por medio de publicidad para orillarlo a su compra.

Palabras clave: Codorniz, Comercializacion, Producción. 


\begin{abstract}
The project aims to determine the demand for quail egg in the city of Tuxpan, Veracruz, which is why it starts with a preliminary observation of the activity in the municipality, through a study of objective type, determined that there is no marketing of the Quail egg It is observed a low habit of consumption of quail egg and therefore it must be made aware of the benefits that this product contributes to the health as well as the price to which it is intended to be so that it penetrates in the Market little by little until we get to position ourselves. In addition, extensive advertising campaigns are required at the local, regional and national levels, so that people consume a high nutritional product and therefore, with great benefits for any type of population, including children, elderly and sick people. All this was determined through a survey applied to the population to achieve our main objective, to determine if there is a demand for the product in the region, concluding that there is indeed a demand since $98 \%$ of the population surveyed answered that You agree to purchase the product as soon as it is offered for sale. This is also due to the fact that most of them do not know the product and therefore are not willing to buy it due to this ignorance, being important to position it in the consumer's mind through publicity to border on their purchase.
\end{abstract}

Keywords: Codorniz, Marketing, Production.

\section{INTRODUCCIÓN}

México es el primer consumidor de huevo a nivel internacional, el consumo per cápita de huevo es de $22.3 \mathrm{~kg}$ (Food and Agriculture Organization FAO, 2016). La producción de huevos es una actividad económica que se desarrolla en prácticamente todos los países del mundo. En las últimas décadas han surgido como potencias productoras China y otros grandes países de Asia, donde se producen más de la mitad de los huevos para el consumo mundial. Su importancia es enorme también en la medida en que el crecimiento de la población en la zona y su desarrollo económico va acompañado de un mayor consumo de alimentos de origen animal, que en esta área se basa esencialmente en huevos y carne de ave (Instituto de estudios del huevo, 2009). En la región se consumen preferentemente los huevos de gallina, pero también los huevos de codorniz, aunque en forma mucho más restringida. En los últimos años ha cobrado difusión el consumir huevos de codorniz ya que su contenido de colesterol es muy poco, así como un mayor valor nutritivo. Él huevo de codorniz es uno de los alimentos más completos para la alimentación humana, pues en su composición figuran proteínas de excelente valor biológico con la mayor parte de los aminoácidos esenciales, además de vitaminas, minerales y ácidos grasos esenciales.

Con respecto a la cotornicultura no hay un desarrollo empresarial o unidades productivas pecuarias importantes en esta región de la economía y de la cadena de los alimentos ya que los productores dedicados a explotar y comercializar los productos derivados de esta especie lo

Revista Científica Biológico Agropecuaria Tuxpan 4 (2) 
hace por pasatiempo y por autoconsumo. La cotornicultura es una rama de la avicultura cuya finalidad es la de criar, mejorar y fomentar la producción de codornices para aprovechar sus productos: huevos, carne, entre otros. La cotornicultura es una alternativa de diversificación generadora de ingreso y empleo rural, contribuyendo, de esta manera, a la reactivación de esta zona; además trae un sin número de ventajas al productor pues en un área mínima de tierra se establece la granja, genera buena cantidad de dividendos con la obtención de los productos y subproductos (Vásquez y Ballesteros, 2007). El huevo es una de las mejores y más económicas fuentes de proteína de alta calidad y contiene un balance equilibrado de los distintos minerales y vitaminas (González y Hernández, 2011). Es importante señalar que en este sector avícola hace falta hacer énfasis sobre el consumo y producción de huevo de codorniz ya que el mercado en el país no es tan grande para este producto, y sólo es consumido por personas que conocen su valor nutricional, sus bajos contenidos de colesterol y alto aporte inmunológico, lo que indica la necesidad de educar a la población consumidora sobre sus beneficios en la salud respecto al

huevo de gallina. La principal problemática de la codorniz es que solo es utilizada para consumo personal o para mascota doméstica y de esta manera no es aprovechada esta ave, que puede ofrecernos múltiples maneras de explotarla correctamente como lo es en la comercialización del huevo.

En la actualidad en la región no hay empresas competidoras que actualmente produzcan y comercialicen huevos de codorniz, no hay competencias debido a que las pocas personas que se dedican a producir huevos de codorniz lo hacen por pasatiempo y para satisfacer las necesidades de auto consumo, lo anterior indica que no se puede hablar de una competencia directa ya que la producción de esta no es destinada para la venta. Los consumidores de huevos de codorniz se encuentran distribuidos en todos los estratos socios económicos, puesto que es un producto altamente nutritivo $y$ balanceado. De manejo sencillo y bajo costo, la codorniz se ha convertido en una herramienta para mejorar la calidad alimenticia de las mesas populares y para generar ganancias considerables a los

productores. Para mejorar la comercialización en el sector se debe realizar una excelente planificación $y$, sobre todo, un estricto control para llevar a cabo la venta, cuidando los factores que intervienen en la misma, todo esto para lograr una comercialización satisfactoria que permita competir en precio y calidad. En base a lo anterior se plantea como objetivo Elaborar un estudio de mercado para identificar si existe demanda de huevo de codorniz para su comercialización, en la región de Tuxpan, Veracruz. 


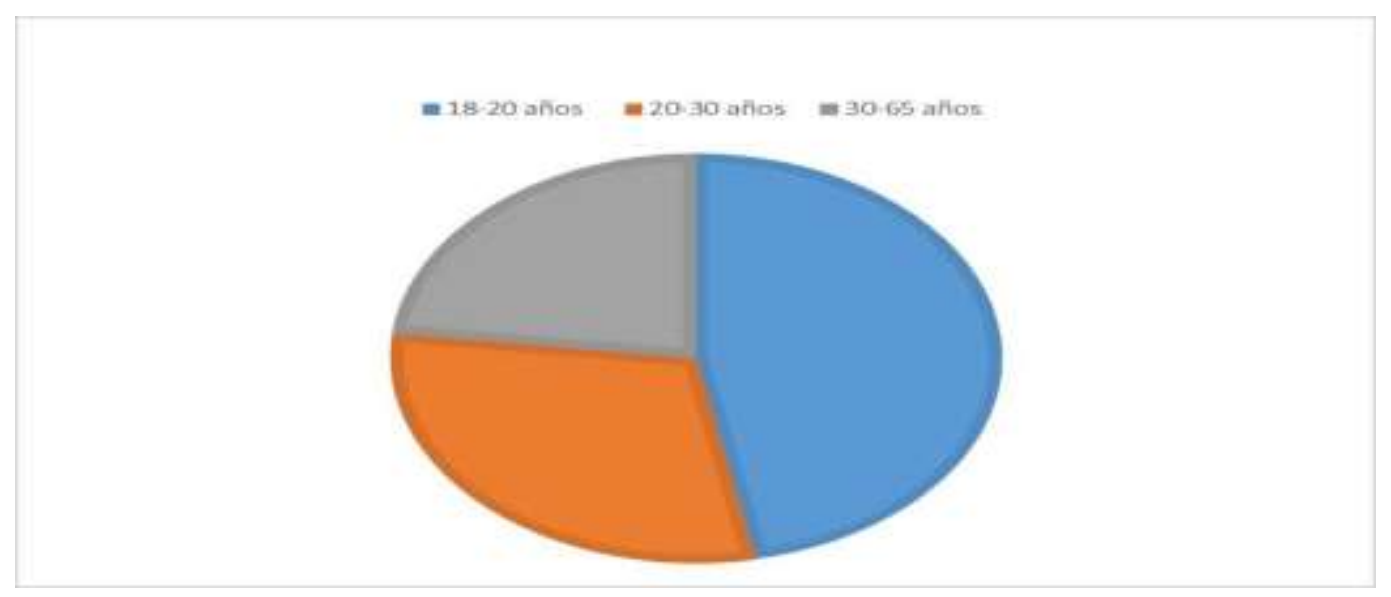

Figura 1. Edad de la población encuestada.

De las 399 personas 390 consumen huevo y solo 9 no, esto hace que más del $90 \%$ de la población consuman huevo, lo que lo hace un mercado totalmente potencial, de igual manera, habría que tener en cuenta que tipo de huevo consumen, ya que la gente consume en su gran mayoría huevo de gallina, que es la principal competencia del huevo de codorniz. El 80\% de la población encuestada no conoce el huevo de codorniz ya que es un producto poco explotado en la región, por lo tanto tenemos la tarea de difundirlo para poder lograr posicionarnos en el mercado y tener una buena demanda del producto. Un $97 \%$ de la población encuestada desconoce acerca de los beneficios del huevo de codorniz, por lo tanto debemos hacer conciencia de estos y aplicar estrategias para promover sus beneficios y sabor ante los clientes potenciales para poder posicionarnos donde queremos. Se observa que la gran mayoría de personas desean encontrar los huevos de codorniz en súper mercados lo que indica que es en estos lugares es donde se debe distribuir la mayor cantidad de el producto. Otro lugar en el cual se podría distribuir los huevos de codorniz es en Oxxo debido a que el producto es catalogado para algunos como una botana y estos lugares son más frecuentados para buscar estos productos.

Como vemos en la grafica las personas que comprarían nuestro producto tienen entre 20 a 50 años de edad. Lo que nos da una aproximación del segmento de mercado al cual nos vamos a dirigir.

Estos resultados muestran que efectivamente existe una demanda del huevo de codorniz del $98 \%$ en la región debido a que un gran porcentaje de la población está dispuesta a adquirir el producto si este es puesto a la venta al público en general, utilizaremos a nuestro favor el desconocimiento de las personas del huevo para que por interés estas compren el producto y de esta manera alcanzar nuestra meta que es introducir el huevo de codorniz al mercado la cual ha estado olvidada como actividad pecuaria en la región ignorando los múltiples beneficios que puede ofrecer como la salud y económicos. Por lo tanto debemos darnos a la tarea de aplicar diferentes estrategias de comercialización para lograr nuestro objetivo, iniciando con dar a conocer el producto y su sabor dando degustaciones en puntos estratégicos, así 
como darlos a conocer con estrategias de mercadotecnia para su publicidad.

Observamos además que la población prefiere un buen precio sin descuidar la calidad y el sabor del mismo. Los huevos de codorniz nos brindan todos lo antes mencionados no siendo su precio un problema debido a que es más barato que el de gallina y de mejor sabor, con mejores atributos alimenticios y de buena calidad y aplicando los mejores estándares de calidad para su producción en la granja donde son producidos, cuidando la alimentación y la tranquilidad de las aves para de esta manera tener un excelente producto final. Se observo que la población consume huevo en grandes cantidades factor de vital importancia para nosotros aunque debemos considerar que el huevo más consumido es el de gallina, nuestra competencia directa.

\section{CONCLUSIONES}

Se determinó una demanda del 98 \% para los huevos y está dispuesto a comprar en cuanto el producto sea puesto a la venta. Sí, se logra abastecer este mercado, de personas de entre 20 y 50 años. La cantidad de familias potenciales dispuestas a consumir los huevos es alta.

La cría de codorniz es una actividad que puede iniciarse con poco dinero. Muchos criadores comienzan su producción con 60 a 800 codornices en lugares poco convencionales.

Algunas ventajas de la cría de codorniz son: Poca competencia, Producción altamente intensiva, Bajo costo de Instalaciones, Los huevos de la codorniz son más ricos en vitaminas y minerales que los de gallina y de mejor sabor, El huevo de codorniz es recomendado por pediatras para la alimentación de niños y ancianos por sus bajos niveles de colesterol y alto contenido proteico, Se cuenta con condiciones climáticas óptimas para realizar la actividad.
La viabilidad del negocio depende en gran medida de la necesidad de ofrecer productos sanos, de calidad, con la debida diversificación y segmentación del mercado, asumiendo un manejo adecuado de los recursos ambientales con el menor uso de insumos químicos, mejorando todos los procesos y buscando producir volúmenes grandes, con productos naturales que no afecten la salud humana. La cría de codorniz es una excelente actividad pecuaria y se puede realizar en la región con la posibilidad de obtener buenos resultados del público en general, y con el apoyo de las distintas dependencias.

\section{LITERATURA CITADA}

Arbaiza, F. Teresa. Agro Enfoque. aug2009, Vol. 23 Issue 165, p6266. 5p. 5 Color Photographs, 4 Charts.

Bazán, Juan Alva. 2007. La codorniz en Perú. Agro Enfoque., Vol. 22 Issue 157, p76-78. 3p.

Cumpa Gavidia, Marcial. 2014. Perspectivas de la Producción de Huevos de Codorniz en el Perú. Revista Agro Enfoque, Vol. 29 Issue 194, p32-35. 4p

Garcés García-Espinosa, Luis, Yoldi Borzhetskaia, Tatiana, Ledesma Rivero, Luis. Sobre los alimentos denominados afrodisiacos. Rev Cub Aliment Nutr 2009;19(2):194-210.

Tapia Robles et al. 2002. Densidad poblacional de la codorniz Moctezuma

(Cyrtonyx montezumae) en la región noroeste del Estado de México, México. Veterinaria México. jul-sep2002, Vol. 33 Issue 3, p255-263. 9p. 
Copyright (c) 2016 Joaquin Eduardo Diaz Reyes, Roberto Alejandro Mateos Rocha, Pablo Elorza Martinez, Nora Garcez Mercado, Edalid Álvarez Velázquez, Sonia Reyes Reyes, Blanca Vianey Hidalgo Barrios y Oralia Elorza Martinez

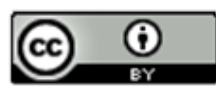

Este tex to está protegido por una licencia licencia Creative Commons 4.0

Usted es libre para Compartir —copiar y redistribuir el material en cualquier medio o form ato-y Adaptar el documento —remezdar, transformar y crear a partir del material- para cualquier propósito, incluso para fines comerciales, siempre que cumpla la condición de:

Atribución: Usted debe dar crédito a la obra original de manera adecuada, proporcionar un enlace a la licencia, e in dicar si se han realizado cambios. Puede hacerlo en cualquier form a razonable, pero no de form a tal que sugiera que tiene el apoyo del licenciante o lo recibe por el uso que hace de la obra.

Resumencielicencia - Textocompletodelalicencia 